Revista Tecné, Episteme y Didaxis: TED. Año 2014, Número Extraordinario. ISSN Impreso: 0121-3814, ISSN web: 2323-0126

Memorias, Sexto Congreso Internacional sobre Formación de Profesores de Ciencias. 08 al 10 de octubre de 2014, Bogotá

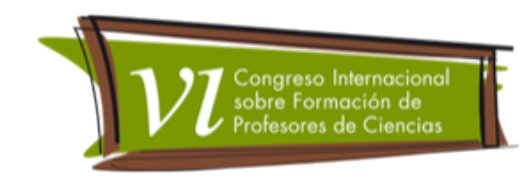

\title{
Relaciones actitudinales de los estudiantes hacia el concepto de circulación en plantas desde el enfoque conflicto cognitivo
}

Cepeda Benavides, Wilson' - Martínez Parra, Miguel Angel²

Categoría 1. Reflexiones y experiencias desde la innovación en el aula.

\section{Resumen}

Este artículo propone exponer los resultados de la implementación de una unidad didáctica diseñada para estudiantes de sexto grado, enmarcada desde el enfoque conflicto cognitivo y respaldado por actividades prácticas en el concepto de circulación en plantas. Se elaboraron preguntas-conflicto suscitando en los educandos bosquejos o esbozos mentales que soportaran una explicación fundamentada en una planta escogida, a medida que se esclarecía la pregunta se presentaba la teoría alterna, y se planteaba una nueva pregunta relacionada con una mayor complejidad. Logrando una apropiación en los estudiantes hacia la ciencia su importancia y aprendizaje, por medio de las preguntas se consiguió que acrecentaran su interés hacia el concepto de circulación, dicho cambio actitudinal es positivo ya que genera en ellos una motivación hacia el aprendizaje.

\section{Palabras claves}

preguntas-conflicto, circulación, plantas, teoría alterna, Motivación.

\section{Marco teórico}

Lo que buscamos con ésta apuesta didáctica es que el estudiante pueda por lo menos utilizar su conocimiento en otro contexto y por qué no, llegar a hacer una integración jerárquica del Sistema Circulatorio (S.C.) con otros sistemas y evitar que el conocimiento previo del estudiante no sea solapado por el científico. Pozo \& Gómez (1998).

Por otra parte en cuanto a las estrategias (Pozo 1998), lo primero que se debe hacer es buscar las ideas previas que tienen los estudiantes, luego presentar de la teoría alterna, hacer una comparación entre las teorías del alumno y las teorías alternas, aplicación de las nuevas teorías a problemas ya explicados por el

\footnotetext{
1 Universidad Distrital Francisco José De Caldas. Bogotá, Colombia; wilcebe90@hotmail.com

2 Universidad Distrital Francisco José De Caldas. Bogotá, Colombia;

mianmapa@gmail.com
} 
Revista Tecné, Episteme y Didaxis: TED. Año 2014, Número Extraordinario. ISSN Impreso: 0121-3814, ISSN web: 2323-0126

Memorias, Sexto Congreso Internacional sobre Formación de Profesores de Ciencias. 08 al 10 de octubre de 2014, Bogotá

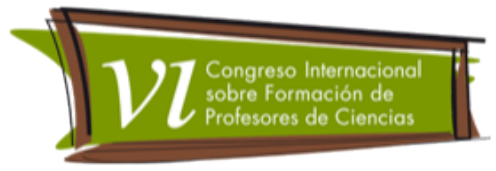

alumno y no explicados y hacer un consenso elaborado para dar explicación a la pregunta de una manera más argumentada. De esta manera funciona el Conflicto Cognitivo (C.C)

Lo actitudinal, se puede ver desde: lo afectivo, lo cual depende de la aceptación, indiferencia o rechazo hacia las ideas o teorías alternas y por otro lado, los esquemas anticipativos y preparativos de una actuación determinada (actitud) (Martin \& Pérez, 2008).

La enseñanza de las ciencias en las escuelas no ha sido acompañada de una curiosidad o aprecio por partes de los estudiantes (Furió, 1994). Las investigaciones han mostrado que el cambio actitudinal de aquellos hacia el aprendizaje de las Ciencias es negativo y este aumenta con los años (Schibeci, 1984). Puesto que los estudiantes no logran tener un consenso entre los contenidos y sus ideas previas, dicha arbitrariedad genera una desmotivación conllevando a una actitud negativa hacia el aprendizaje de la ciencia.

Estas actitudes negativas ocasionan una grave ruptura en el rol del estudiante, donde se desarticula de las diversas dinámicas propuestas para su enseñanza, esto se puede atribuir a la escuela ya que se enseña verazmente un contenido el cual es insuficiente para el estudiante para emplearlo en su contexto. (Furió, 1994).

El maestro reduce las actividades académicas a ejercicios de receta. Presentando una ciencia que se enfoca en problemas y en ejercicios de memoria que no encajan con los problemas reales que los rodea ni los resuelve (cené, 1992)

\section{Metodología}

La metodología de este trabajo se enmarca en el paradigma interpretativo, (Kuhn, 1980). Mediante el diseño y la implementación de una unidad didáctica la cual se realizó bajo el enfoque del C.C.

El diseñó de la unidad didáctica se realizó con respecto al concepto de circulación, donde se tuvo en cuenta los estándares del Ministerio de Educación Nacional (M.E.N.) Y los del Instituto Colombiano del Fomento a la Educación Superior (I.C.F.E.S.) Además de una revisión conceptual del concepto, Se planeó seis clases en la cuales se trabajó alrededor del concepto de circulación en plantas. 
Revista Tecné, Episteme y Didaxis: TED. Año 2014, Número Extraordinario. ISSN Impreso: 0121-3814, ISSN web: 2323-0126

Memorias, Sexto Congreso Internacional sobre Formación de Profesores de Ciencias. 08 al 10 de octubre de 2014, Bogotá

La implementación se llevó a cabo en la Institución Educativa Distrital (I.E.D.) Policarpa Salavarrieta, en la ciudad de Bogotá, en el grado 604, el cual estaba con formado por un numero de 30 estudiantes, con edades que oscilaban entre los 11 y los 15 años.

Tabla 1. Se indica los temas abordados en cada sesión de trabajo con los estudiantes. Las $x$ indican el lugar de la actividad.

\begin{tabular}{|c|c|c|c|c|c|}
\hline \multirow{2}{*}{$\begin{array}{l}\text { \# Sesión } \\
\text { de clase }\end{array}$} & \multirow[t]{2}{*}{ Tema } & \multicolumn{3}{|c|}{ Actividad } & \multirow[t]{2}{*}{ Pregunta problema } \\
\hline & & tipo & $\begin{array}{l}\text { Fuera } \\
\text { del } \\
\text { salón }\end{array}$ & $\begin{array}{l}\text { Dentro del } \\
\text { salón }\end{array}$ & \\
\hline 1 & S.C. & $\begin{array}{l}\text { Definir preguntas con } \\
\text { respecto al S.C. }\end{array}$ & & & 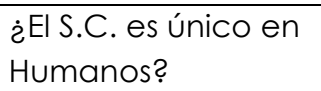 \\
\hline 2 & Sangre & $\begin{array}{l}\text { Relacionar diversos } \\
\text { organismos que } \\
\text { presentan sangre }\end{array}$ & & $X$ & $\begin{array}{l}\text { ¿Solo la sangre } \\
\text { constituye el S.C.? }\end{array}$ \\
\hline 3 & $\begin{array}{l}\text { Circulación en } \\
\text { las plantas }\end{array}$ & $\begin{array}{l}\text { Observar diversas } \\
\text { plantas explicar de } \\
\text { qué manera funciona } \\
\text { su sistema }\end{array}$ & $x$ & & $\begin{array}{l}\text { ¿De qué forma es la } \\
\text { circulación en } \\
\text { plantas? }\end{array}$ \\
\hline 4 & $\begin{array}{l}\text { S.C. en } \\
\text { invertebrados }\end{array}$ & $\begin{array}{l}\text { Dibujar y explicar el } \\
\text { S.C. de invertebrados }\end{array}$ & & $x$ & $\begin{array}{l}\text { ¿Qué tipo de } \\
\text { circulación hay en } \\
\text { los diversos } \\
\text { invertebrados? }\end{array}$ \\
\hline 5 & $\begin{array}{l}\text { S.C. en } \\
\text { Vertebrados }\end{array}$ & $\begin{array}{l}\text { Realizar actividad } \\
\text { física, explicar la } \\
\text { relación del S.C. con } \\
\text { los otros sistemas }\end{array}$ & $x$ & & 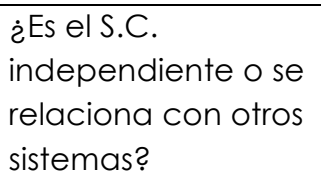 \\
\hline 6 & $\begin{array}{l}\text { integración } \\
\text { sistema -sistema } \\
\text { a un contexto } \\
\text { nutrición y } \\
\text { obtención de } \\
\text { energía }\end{array}$ & $\begin{array}{l}\text { Laboratorio, colocar } \\
\text { un clavel en tinta } \\
\text { china durante ocho } \\
\text { días, explicar que } \\
\text { procesos ocurren en la } \\
\text { tinción del clavel }\end{array}$ & & $x$ & $\begin{array}{l}\text { ¿Por qué la } \\
\text { coloración del } \\
\text { clavel varia al } \\
\text { colocarla en tinta } \\
\text { china? }\end{array}$ \\
\hline
\end{tabular}


Revista Tecné, Episteme y Didaxis: TED. Año 2014, Número Extraordinario. ISSN Impreso: 0121-3814, ISSN web: 2323-0126

Memorias, Sexto Congreso Internacional sobre Formación de Profesores de Ciencias. 08 al 10 de octubre de 2014, Bogotá

Se realizó seguimiento a seis estudiantes, se utilizó un diario de campo donde se anotó las actitudes de los estudiantes con respecto a cada una de las clases, se recogieron todos los trabajos ilustrativos y algunas clases donde los estudiantes argumentaban verbalmente se grabaron (sesión 2, 3 y 6), permitiendo así dar un mayor soporte al cambio conceptual producto de su nueva actitud hacia los temas.

Al comenzar cada uno de los temas, se les pedía a los estudiantes elaborar un dibujo acerca del concepto que se iba a abordar; para revisar sus ideas previas que son una pieza clave para el enfoque (C.C.). Se diseñaron tres clases dentro del salón y tres fuera del, con el objetivo de observar como los entornos físicos pueden afectar las actitudes de los estudiantes para comprender un concepto.

\section{Resultados}

Los resultados que a continuación se muestran son fragmentos de algunas sesiones de clase en las cuales se reflejó de qué manera el C.C. contribuyó en la comprensión del concepto de circulación en plantas.

Figura 1: ésta gráfica representa el equilibrio y desequilibrio antes y después de presentar una situación conflicto. El estudiante se desenvuelve entre sus ideas previas, sus argumentos científicos y entre los constructos de estos dos hechos con el maestro. Cuando se le presenta una situación problema, sus ideas previas pueden estar o no, susceptibles al cambio, al presentarle la idea alterna puede acontecerle que: se llegue a un pacto entre teoría alterna-idea previa (equilibrio) y se pase a una situación más compleja; se esté insatisfecho y se requiera de más teorías alternas hasta que se logre un equilibrio entre estas. Cual sea el caso y a medida que se avance en el concepto, siempre se estará experimentando una situación equilibrio-desequilibrio enmarcado en los consensos elaborados. Las palancas motivacionales impiden que el estudiando permanezca siempre en un estado de desequilibrio permitiendo que él sienta la necesidad de encontrar una respuesta que lo lleva nuevamente a un estado de equilibrio. 
Revista Tecné, Episteme y Didaxis: TED. Año 2014, Número Extraordinario. ISSN Impreso: 0121-3814, ISSN web: 2323-0126

Memorias, Sexto Congreso Internacional sobre Formación de Profesores de Ciencias. 08 al 10 de octubre de 2014, Bogotá

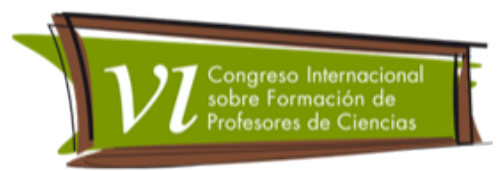

Figura 1. Punto de equilibrio

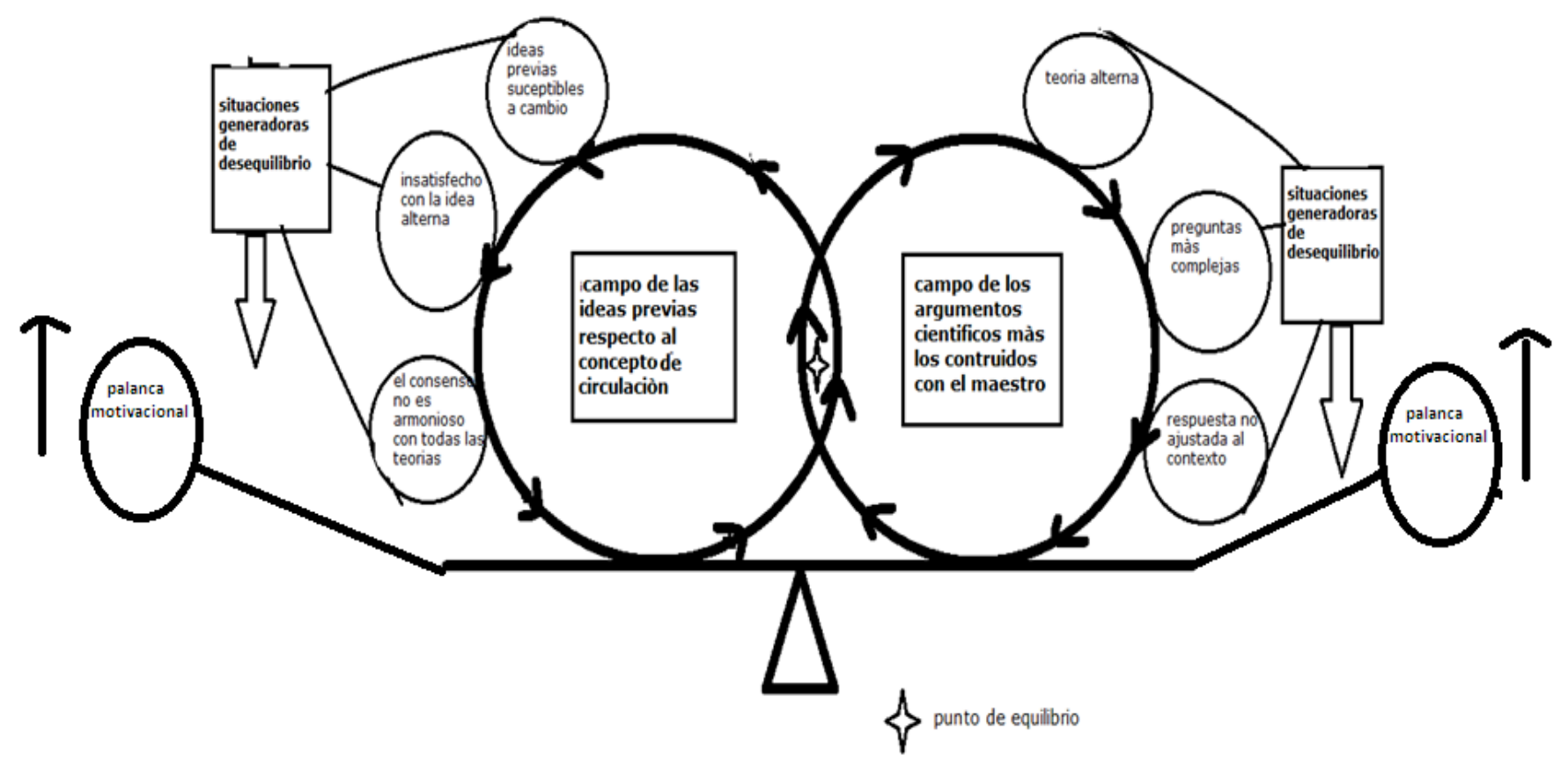

En una de las grabaciones realizadas en la sesión dos, se evidencia como la estudiante María Moreno entra en equilibrio y desequilibrio, está en equilibrio

cuando se afianza de que las plantas tienen sangre, se desequilibra al pensar en la pregunta alterna ¿ Cómo es la "sangre" de las plantas?), la estudiante ahora se encuentra insaciable al pensar en el vacío que deja su concepto (sangre) dentro de la planta, otro estudiante presenta su argumento más elaborado y suscita a la estudiante a consensar entre lo dicho por ella, su compañero y el profesor para elaborar su respuesta, dicho consenso - Ver tabla 2 (Columna 4 - Fila 2) - motiva al estudiante a buscar afanosamente solución a esa incógnita. Reiterando Pozo (1997). Al enfrentar al estudiante a este tipo de situaciones- problema le genera un desequilibrio, permitiendo el avance de un concepto. Pero no solo eso, También podemos aludir a Moya, \& Campanario (1999) quienes problematizan que al presentarles situaciones inconsistentes, "el estudiante puede elegir entre admitir las dos versiones como correctas cada una dentro de su propio dominio o rechazar una de las dos". En este caso María Moreno, rechazó la de sangre pero no para abolirla de sus ideas previas sino para utilizarla en otro contexto, según el caso, el estudiante puede ser consecuente del proceso de comprensión- 
Revista Tecné, Episteme y Didaxis: TED. Año 2014, Número Extraordinario. ISSN Impreso: 0121-3814, ISSN web: 2323-0126

Memorias, Sexto Congreso Internacional sobre Formación de Profesores de Ciencias. 08 al 10 de octubre de 2014, Bogotá

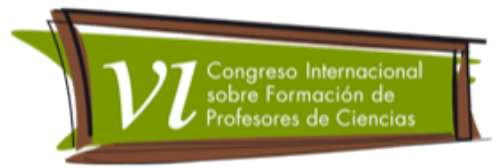

construcción y avance del concepto de ser así, se incita en ellos un estado de metacognición.

Tabla 2. Se muestra las etapas como se dio el proceso de aprendizaje durante las sesiones 2, 3 y 6 , teniendo en cuenta las ideas previas de los estudiantes, se generó una idea alternativa al fenómeno en estudio, con respecto a estas dos ideas los estudiantes generan una concepción más clara alcanzada en consenso.

\begin{tabular}{|c|c|c|c|}
\hline Pregunta & Idea previa & Idea alterna & Consenso \\
\hline $\begin{array}{l}\text { ¿́Las plantas tienen } \\
\text { circulación? }\end{array}$ & $\begin{array}{l}\text { "Tiene que absorber } \\
\text { agua, nutrientes y savia } \\
\text { para no morir" }\end{array}$ & $\begin{array}{l}\text { Conceptualización } \\
\text { acerca del S.C. }\end{array}$ & $\begin{array}{l}\text { "el S.C. ayudaba a la } \\
\text { nutrición de la planta y } \\
\text { que la savia es una } \\
\text { mezcla de lo que la } \\
\text { planta absorbe" }\end{array}$ \\
\hline $\begin{array}{l}\text { ¿Las plantas tienen } \\
\text { sangre? }\end{array}$ & $\begin{array}{l}\text { "Si tienen sangre, pero } \\
\text { que es blanca o } \\
\text { trasparente, la sangre es } \\
\text { el agua" }\end{array}$ & $\begin{array}{l}\text { Conceptualización } \\
\text { acerca de la absorción } \\
\text { de nutrientes }\end{array}$ & $\begin{array}{l}\text { "la planta no tiene } \\
\text { sangre, ésta la } \\
\text { presentan los animales, } \\
\text { lo que circula por la } \\
\text { planta es su alimento } \\
\text { para nutrirse que viene } \\
\text { de las raíces y de las } \\
\text { hojas" }\end{array}$ \\
\hline $\begin{array}{l}\text { ¿Cómo sube el } \\
\text { agua y nutrientes si } \\
\text { no tienen corazón? }\end{array}$ & $\begin{array}{l}\text { "Las plantas no tienen } \\
\text { corazón", }\end{array}$ & $\begin{array}{l}\text { Conceptualización } \\
\text { capilaridad, aclarando } \\
\text { el ejemplo del "pitillo" } \\
\text { de un estudiante }\end{array}$ & $\begin{array}{l}\text { "La capilaridad ayuda } \\
\text { a que la planta suba los } \\
\text { nutrientes por el tallo } \\
\text { hasta las hojas y de ahí } \\
\text { se distribuya para todas } \\
\text { partes" }\end{array}$ \\
\hline
\end{tabular}

Además de una concepción alcanzada en consenso, los estudiantes manifiestan un agrado y satisfacción por apropiarse de su proceso de aprendizaje, es decir, las preguntas conflicto les genera un aislamiento de las clases tradicionales y los lleva a un lugar donde ellos se sienten más cómodos, en un estado de equilibriodesequilibrio, apuntando que los espacios físicos como los laboratorios permitían dinamizar más los conceptos, encontrando que los estudiantes no sienten apatía por las preguntas y mucho menos por los conceptos o por la biología, como lo que encontró Melo, A. \& Jiménez, S. (2013) a comenzar sus clases les pregunta ¿Qué pregunta quisieras que el profesor te respondiera en clase de biología? los estudiantes tenían respuestas apáticas como: "No sé"; "Nada, lo que me enseñe está bien"; "No quisiera ver ningún tema". Bajo el enfoque de resolución de problemas y un proceso motivacional con procesos lúdicos fuera del salón logró 
Revista Tecné, Episteme y Didaxis: TED. Año 2014, Número Extraordinario. ISSN Impreso: 0121-3814, ISSN web: 2323-0126

Memorias, Sexto Congreso Internacional sobre Formación de Profesores de Ciencias. 08 al 10 de octubre de 2014, Bogotá

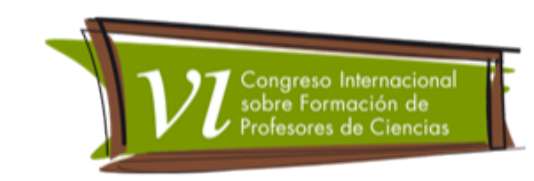

en su última sesión un cambio total cuando varios estudiantes comentaron: "me gustaría estudiar insectos en biología porque en eso me puede servir para criminalística que es lo que voy a estudiar cuando salga"

\section{Conclusiones}

El C.C. es un enfoque el cual permite que el estudiante construya su propio conocimiento, donde el docente se centra en preguntas problemas las cuales generan un desequilibrio en el conocimiento del estudiante, para realizar estas preguntas se debe tener en cuenta las ideas previas que tiene el estudiante, ya que son estas las que orientarán el proceso de aprendizaje en los estudiantes, planteando dinámicas en clase acordes a las expectativas del estudiantado.

Es de vital importancia tener en cuenta la satisfacción y motivación del estudiante respecto a lo aprendido en cada sesión de clase, puesto que con esta información se replanteará la siguiente sesión de clase, con el fin de generar un desequilibrio conceptual, para lograr dicho desequilibrio se tuvo en cuenta tres aspectos indispensables que fueron; ideas previas, Ideas alternativas y la idea llegada en el consenso, esta última fue la que encaminó la planeación de actividades.

\section{Referencias bibliográficas}

Cené, A. (1992): "La historia de la Ciencia, hilo conductor de la enseñanza y el aprendizaje." Aula, 4/5, pág. 22-28.

Furió Más, C. J. (1994). Unas relaciones afectivas controvertidas: las actitudes de los estudiantes hacía las ciencias y su aprendizaje. Aula de Innovación Educativa, 2(27), pág. 5-8.

Kunn, T. S. (1980). Los paradigmas científicos. En Estudios sobre sociología de la ciencia (pág. 79-102). Alianza Editorial.

Martín, L. \& Pérez, R. (2008) Estructuras conceptuales, metodológicas, actitudinales de estudiantes de una institución educativa rural. Studiositas, edición de diciembre de 2008, vol. 3, núm. 3

Melo, A. G., \& Jiménez, S. R. (2013) cambio actitudinal de estudiantes de octavo grado hacia el aprendizaje de interacciones biológicas mediante la resolución de problemas. IX Congreso Internacional Sobre Investigación en Didáctica de las Ciencias: pág. 1478-1482 
Revista Tecné, Episteme y Didaxis: TED. Año 2014, Número

Extraordinario. ISSN Impreso: 0121-3814, ISSN web: 2323-0126

Memorias, Sexto Congreso Internacional sobre Formación de Profesores de Ciencias. 08 al 10 de octubre de 2014, Bogotá

Moya, A., \& Campanario, J. M. (1999). ¿Cómo enseñar ciencias? Principales tendencias y propuestas. Enseñanza de las ciencias: revista de investigación y experiencias didácticas, 17(2), pág. 179-192.

Pozo, J. I., \& Gómez, M. A. (1998). Aprender y enseñar ciencia: del conocimiento cotidiano al conocimiento científico. Madrid. Ediciones Morata.

Pozo, J.I. (1997).Teorías cognitivas del aprendizaje. Madrid. Ediciones Morata.

Schibeci, R.A. (1986): "Attitudes to science: an update." Studies in Science Education, 11, pág. 26-59 